\title{
MANUFACTURING AND THE RISE OF ARTIFICIAL INTELLIGENCE: INNOVATION CHALLENGES
}

\author{
Zeljko Tekic, Ilija Cosic \& Branko Katalinic
}

Skolkovo Institute of Science and Technology, Moscow, Russia; University of Novi Sad, Novi Sad, Serbia; Vienna University of Technology, Vienna, Austria
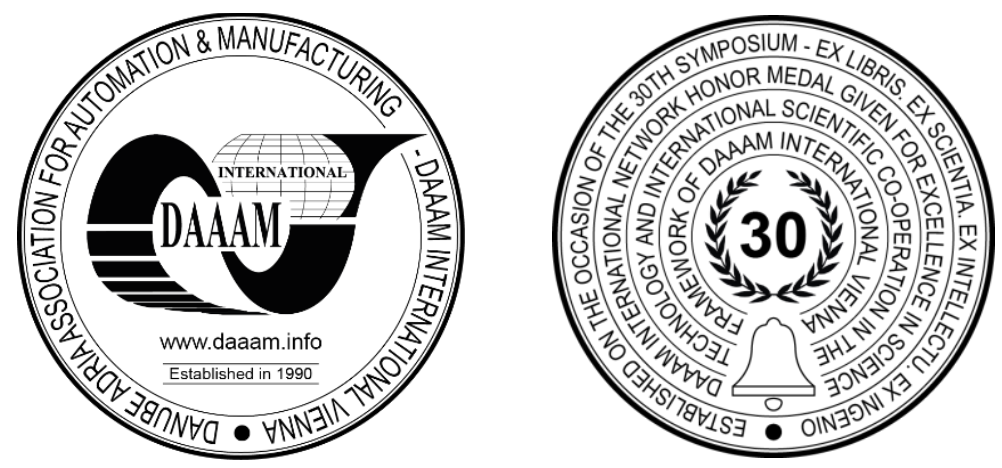

This Publication has to be referred as: Tekic, Z[eljko]; Cosic, I[lija] \& Katalinic, B[ranko] (2019). Manufacturing and the Rise of Artificial Intelligence: Innovation Challenges, Proceedings of the 30th DAAAM International Symposium, pp.0192-0196, B. Katalinic (Ed.), Published by DAAAM International, ISBN 978-3-902734-22-8, ISSN 1726-9679, Vienna, Austria

DOI: $10.2507 / 30$ th.daaam.proceedings.025

\begin{abstract}
The peak of the main wave of artificial intelligence (AI) tsunami is about to hit industries, governments and societies accelerating the pace of product and service innovation, and putting data in the focus of the business interest. AI, as a core driver of the digital revolution, is expected to challenge and transform some of the core axioms and assumptions that underlie the innovation process and its management. To contribute to a better understanding what and how is changing in manufacturing in the era of AI, in this paper we describe five innovation challenges that will, in our view, shape the future of manufacturing. Focusing on changes in innovation focus, importance of data, need for increased collaboration, impact of customer experience and shortage of talent, we offer valuable insights on how manufacturing companies should approach innovation challenges in the next decade.
\end{abstract}

Keywords: manufacturing; artificial intelligence; AI; challenges; innovation.

\section{Introduction}

Artificial intelligence (AI) is the most important general-purpose technology of our times [1]. It is getting used as an input by many downstream sectors [2] enabling innovations in rapidly growing number of products, services and processes. For some industries, AI is becoming a commonplace. For example [3] - it is routinely used by Amazon (e.g. in supply chain solutions - Kiva robot automation in retail logistics), Netflix (e.g. in recommendation systems that target individual customers) and Google (e.g. in autonomous cars). Although the first association for AI based systems for many will be virtual assistants Alexa and Siri, high-speed dynamic pricing of stocks or latest mobile applications, like FaceApp, AI is entering all sectors, including manufacturing, oil and gas, and number of other industries with heavy machinery promising to benefit their operations.

Paired with other key digital technologies, like big data analytics, cloud computing, IoT, robotics and additive manufacturing, AI is expected to change production and manufacturing in the same way as steam machine or conveyor- 
belt assembly lines changed it decades ago. In that paradigm shift, AI solutions, among other things, should allow monitoring, recording and analysing everything in manufacturing process to enable detecting and solving problems, including those that people were not even aware of; make supply chains smart; improve safety performance and quality standards; and provide new and better materials. Bearing in mind that only unplanned downtime caused by equipment failure costs industrial manufacturers more than $\$ 20$ billion annually [4], the benefits AI promises to bring are significant. It is not surprising that in a recent survey $\mathrm{AI}$ is characterised by almost half respondents from the automotive and manufacturing sectors as "highly important" and "absolutely critical to success" to the manufacturing function in the next five years [5]. Or prediction that by the end of 2021, 50\% of all manufacturing companies will be using AI in some form [6]. The measure of this interest is the value of the AI in manufacturing market, that is estimated at USD 1 billion in 2018 and expected to reach USD 17.2 billion by 2025 [7].

$\mathrm{AI}$, as a key driver of the digital revolution, is expected to challenge and transform some of the core axioms and assumptions that underlie the innovation process and its management. To contribute to a better understanding what and how is changing in manufacturing with the rise of AI, in this paper we describe five innovation challenges that will, in our view, shape the future of manufacturing over the next decade. The purpose of this paper is not to give the final list of challenges, but to initiate discussion about this important topic. To do that we start with clarifying what AI can and cannot do, and then continue describing five innovation challenges manufacturing companies are facing in the era of AI.

\section{What AI can and cannot do?}

Artificial intelligence is bringing significant change, but it has been also over-hyped by media, typically due to the confusion between what is possible and what the 'holy grail' of AI - Artificial General Intelligence - may promise to be done in the (undefined) future. So, it is useful, before proceeding to further discussion, to clarify what AI, and especially machine learning as its most promising representative today, can and cannot do at the moment and in a time horizon of 10 years.

Processes, business and manufacturing, are composed of numerous interrelated tasks. Some of these tasks are more, some less suitable for applying AI, while for some tasks AI solutions are completely non-applicable (today or maybe forever). For a company to take advantage of AI and profit from its application, it has to narrow down the tasks where AI can offer advantage over other solutions and organize their execution.

AI can transform and improve performance in the range of tasks where something should be predicted or where task can be reduced to a prediction problem [8]. To be good in predicting, AI needs data about previous behaviour of the system to learn from it. Then, based on the learning it becomes able to predict outcomes of the system from any new input. To be successful, AI needs big and 'good' data for training; without that, typically, accuracy is low and learning slow.

AI is more suited to transform 'one-second thinking' tasks, and much less suited for 'Sherlock Holmes thinking' tasks. $\mathrm{Ng}$ [9] suggests that specific tasks can probably be automated using AI (immediately or in the near future) if a typical person can do it in less than one second of thought. On the other hand, when a task is inherently open-ended, when it requires long chains of logic or reasoning that depend on diverse background knowledge, intuition or common sense to be solved - AI is much less effective [1]. Solving this type of tasks requires what we call here 'Sherlock Holmes thinking'. For example, new opportunity identification asks for 'Sherlock Holmes thinking', as it is based on deep interaction with people, needs different approaches every time it has to be done, and depends on intuition and connecting events over long time horizon. Nevertheless, AI may also give assistance to such tasks by structuring, clustering and condensing rich information, providing guidance and inspiration, and at the same time reducing preparation efforts (Table 1).

\begin{tabular}{|c|c|c|}
\hline & Big and good data available & No or small data available \\
\hline $\begin{array}{l}\text { One-second } \\
\text { thinking task }\end{array}$ & $\begin{array}{c}\text { AI } \\
\text { AI replaces humans }\end{array}$ & $\begin{array}{l}\text { AI + People } \\
\text { AI amplifies human abilities on short-term; on } \\
\text { long-term, as datasets are getting available, AI } \\
\text { replaces humans }\end{array}$ \\
\hline $\begin{array}{l}\text { Sherlock Holmes } \\
\text { thinking task }\end{array}$ & $\begin{array}{l}\text { People }+ \text { AI } \\
\text { AI amplifies human abilities immediately, } \\
\text { but with small chances to replace humans, } \\
\text { even on long-term }\end{array}$ & $\begin{array}{c}\text { People } \\
\text { AI amplifies human abilities, increasing its } \\
\text { contribution over time, but main job on this type } \\
\text { of tasks will be done by humans }\end{array}$ \\
\hline
\end{tabular}

Table 1. What tasks and how AI will transform in the near future 


\section{Innovation challenges}

Focusing on changes in innovation focus, importance of data, need for increased collaboration, impact of customer experience and shortage of talent, we offer valuable insights on how manufacturing companies should approach innovation challenges.

\subsection{From incremental to radical innovation}

Incremental innovation builds on existing practices and exploits existing tech to serve existing customers. Exactly these characteristics (available data and well-known set of rules) favor application of AI. Thus, AI will accelerate incremental improvements. At the same time, AI may make reverse engineering easier and facilitate imitation of leading products and technologies [10]. In the both cases, AI will lead to diminishing returns on investing in incremental innovation and motivate innovators to invest more in substantially new products and services to win over competition. Focus on radical innovation will not be just result of decreasing profits from incremental innovation due to AI, but because AI allows radically new offerings as autonomous driving or individualized medical therapies. Changing the focus from incremental to radical, will ask for significant changes in companies as the most of established companies know how to manage incremental improvements (and spend 80-90\% of technology budgets on them), but have weak understanding of breakthrough innovations [11].

In AI-powered reality, the innovation game brings new rules, including more players and more complexity. In that setting, the ability to design novel business models will become even more crucial. On one hand, this is dictated by increased complexity in which companies, to be successful, will have to figure out how to harmonize changes coming from innovation dependence on data. On the other hand, the role of business model innovation is amplified because of the fact that tasks most suitable to be substituted by AI may be completely different from those that were primary target of automation in the previous technology waves [1]. Thus, becoming an AI company will require creative and blue-sky thinking in understanding core logic of tasks, sometimes even redefining them in a way that can enable full power of AI application [9]. This will typically lead to numerous possibilities for business model and process innovations, as many tasks will be "reinvented" and offering ground for new compositions.

\subsection{Data over patents}

Although AI-related patenting is growing rapidly, with over half of 340,000 identified inventions being published since 2013 [2], innovation success and usage of AI depends primary on having access to (training) data, not patents. Next to many of AI software being open source, most of solutions need to be fed with millions of data points. Thus, data, or more correctly, access to data is the key for innovating in AI era. Bearing that in mind, both strategic planning and implementation should focus on identifying and acquiring right data in large volume. Identifying valuable data is complex task. People with diverse backgrounds and roles in a team should come together in early phase of data acquisition process to define and prioritize types of data to be acquire and saved as well as to plan where, how, when and for how long. And not only that. Significant number of existing "dumb" machines will need first to be smartened by adding sensors and enabling data collection from them (and data exchange with smart machines). So, it is not only about collecting data, but about providing backward compatibility of legacy machines using smart sensors that collect comprehensive data in real time, and transform isolated solutions (machines and tools) into part of network [12].

With regards to IP protection, trade secret is appropriate for a wide scope of AI value chain elements (e.g. AI-generated code, neural networks, or training data) as it offers protection and avoids disclosure. However, it does not exclude others from using same or very similar solutions and thus protection is incomplete. New IP strategies and data disclosures approaches need to be explored.

\subsection{Customer experience}

Individual customer experiences $(\mathrm{N}=1)$ becomes the most important focus point for many companies. Thus, they more and more make specific products, in line with the customer's individual wishes. This requires that companies have resilient, dynamic and flexible business processes and ability to customize for and co-create products with users and customers [13].

There are numerous examples of customization in car and textile industries. And, that is not new. However, what is new is that over the last few years even very complex systems such as the Mars rover Curiosity and the Red Bull's Formula 1 racing car were manufactured in a batch size of one [14]. Additive manufacturing is emerging as a reliable solution for reducing downtime and optimizing supply chain when it comes to complex and customized spare parts. For example, GE Oil \& Gas [15] started to laser scan every part of tools of interest, create a base of digital 3D models which are then, when needed, used in advanced 3D printing methods to print failed parts, reducing down time - from months to an average of three weeks. Also there are companies like Local Motors (www.localmotors.com) that manufactures lowvolume open-source motor vehicle using multiple microfactories.

Flexibility and high responsiveness to customer requests and focus on manufacturing small batches of products will result in factories and machines, which will have to be very differently organized and interconnected. The concept of "plug \& produce" will dominate. It will influence high-level compatibility between machines from different 
manufacturers in order to enable effective and efficient manufacturing of customized products. At the same time, a need for quick and flexible machinery retooling will emerge.

In this context, $\mathrm{AI}$ is expected to make special impact in need and trend identification, concept testing, and technology scouting [16]. AI solutions inherently learn and improve by handling previous cases continuously increasing performances and customer satisfaction. However, companies should not forget to build communities and engage users as it will additionally help them to discover trends and unique opportunities and enable the company to engage in product co-creation with their consumer base.

\subsection{Open innovation ready}

Development of AI has been rooted in academia and, until recently, developed almost without any business influences. Universities have been a leading force in AI research for decades establishing community of kindred spirits and culture of free sharing (e.g. GitHub) and open publishing (e.g. arXiv). Although companies gradually overtook the leading position in AI research [2], culture routed in open and free access to knowledge is so strong that a company must acknowledge it to succeed in AI development. Also, for a number of applications companies will have to partner to access data needed for powerful AI applications. These partners will be diverse - governmental and non-governmental, large and small entities, established and startups - ranging from universities, hospitals and suppliers to platforms, or even competitors.

Succeeding in AI-powered world, means succeeding in open innovation world. Leading manufacturing companies are increasingly aware [17] that they need to explore and exploit both internal and external knowledge sources to accelerate innovation, since majority of them struggle with a lack of resources, access to data and know-how necessary to realize all innovative AI-driven projects. They need to plan co-creation activities across product life cycle [18] to allow inflow of knowledge through organizational boundaries and benefit from it.

Bearing this in mind, companies should work on building and/or contributing to an ecosystem they want to work in by enabling and establishing numerous communication links, promoting active participation of their employees in meetups and conferences, and engaging in exchange with the environment as frequently as possible. This is a challenging task and should start with creating a culture that confronts not-invented-here syndrome and values outside competence and know-how. Typically, it is influenced via incentives, clear communication, project decision criteria, supplier evaluation lists and so on [19].

\subsection{Battle for talent}

Finding and retaining the right talent for innovation in the era of AI is among the most challenging task. Especially, for industrial manufacturing companies. Why? They have to compete with cool startups from across the globe and tech giants like Google, Tencent and Amazon, over the same talent. And that is not easy neither cheap task.

According to the study from 2017, worldwide market needs 2.3 times more than currently available 300,000 "AI researchers and practitioners" [20]. The outcome is obvious - a war for AI talent and skyrocketing salaries in the sector. For example, when Uber opened the Advanced Technologies Center in Pittsburgh, at the beginning of 2015, they hired a group of some 40 employees from the National Robotics Engineering Center at Carnegie Mellon University [21]. Among these 40 people, several were longtime senior lab members. Manufacturing companies will need to make sure they are well-positioned to compete with this type of transfers. Again, the shortage of talent requires companies to become more open towards environment, and to reinvent the collaboration with startups and universities as well as to provide attractive work-conditions and offerings.

\section{Conclusion}

The peak of the main wave of AI tsunami is about to hit industries, governments and societies accelerating the pace of product and service innovation, and putting data in the focus of the business interest. AI, as a core driver of the digital revolution, is expected to challenge and transform some of the core axioms and assumptions that underlie the innovation process and its management. To contribute to a better understanding what and how is changing in innovation across manufacturing companies with the rise of AI, in this paper we describe five innovation challenges that will, in our view, shape the future of manufacturing over the next decade. Focusing on changes in innovation focus, importance of data, need for increased collaboration, impact of customer experience and shortage of talent we offer valuable insights on how manufacturing companies should approach innovation challenges in the era of AI. This list of trends does not pretend to be final, but to describe a playing field and open discussion.

\section{References}

[1] E. Brynjolfsson and T. Mitchell, "What can machine learning do? Workforce implications," Science (80-. )., vol. 358, no. 6370, pp. 1530-1534, 2017.

[2] WIPO, “WIPO Technology Trends 2019: Artificial Intelligence,” Geneva, Switzerland, 2019. 
[3] McKinsey Global Institute, "Notes from the AI frontier: Modeling the impact of AI on the world economy," Discuss. Pap., vol. September, 2018.

[4] WSJ, "Unlocking performance: How manufacturers can achieve top quartile performance," Wall Street Journal, 2017. [Online]. Available: https://partners.wsj.com/emerson/unlocking-performance/how-manufacturers-canachieve-top-quartile-performance/. [Accessed: 30-Aug-2019].

[5] Forbes, "How AI builds a better manufacturing process," Forbes Insights, 2018. [Online]. Available: https://www.forbes.com/sites/insights-intelai/2018/07/17/how-ai-builds-a-better-manufacturingprocess/\#1 aa430711e84. [Accessed: 30-Aug-2019].

[6] A. Bourne, "What's Ahead for Manufacturing AI," Industry Week, 2019. [Online]. Available: https://www.industryweek.com/technology-and-iiot/what-s-ahead-manufacturing-ai. [Accessed: 30-Aug-2019].

[7] Markets and Markets, "Artificial Intelligence in Manufacturing," 2019. .

[8] A. Agrawal, J. Gans, and A. Goldfarb, "How AI will change the way we make decisions," Harv. Bus. Rev., 2017.

[9] A. Ng, "What artificial intelligence can and can't do right now," Harv. Bus. Rev., vol. 9, 2016.

[10] P. Aghion, B. F. Jones, and C. I. Jones, “Artificial intelligence and economic growth,” No. w23928, 2017.

[11] E. B. Roberts, "Managing invention and innovation,” Res. Manag., vol. 50, no. 1, pp. 35-54, 2007.

[12] B. Katalinic, "Engineers for Knowledge Based Society," in Annals of DAAAM for 2010 \& Proceedings of the 21th International DAAAM Symposium, 2010.

[13] C. K. Prahalad and M. S. Krishnan, The New Age of Innovation. 2008.

[14] K. Nikolaus, "Manufacturing: Self-Organizing Factories," Siemens - Digital Factories, 2014. [Online]. Available: http://www.siemens.com/innovation/en/home/pictures-of-the-future/industry-and-automation/digtial-factorytrends-industry-4-0.html.

[15] GE, "GE Oil \& Gas starts strong in 2017: Innovative digital customer agreements," 2017.

[16] HYVE, “Autonomous Innovation - How AI and Algorithm revolutionize Innovation Management," 2019.

[17] PWC, "Rethinking innovation in industrial manufacturing," 2013.

[18] A. Orcik, Z. Tekic, and Z. Anisic, “Customer co-creation throughout the product life cycle,” Int. J. Ind. Eng. Manag., vol. 4, no. 1, pp. 43-49, 2013.

[19] O. Gassmann, E. Enkel, and H. Chesbrough, "The future of open innovation,” R D Manag., vol. 40, no. 3, pp. 213221, 2010.

[20] Tencent Research Institute, “Global AI Talent White Paper,” 2017.

[21] C. Thompson, "Uber Would Like to Buy Your Robotics Department," The New York Times Magazine, 2015. [Online]. Available: http://www.nytimes.com/2015/09/13/magazine/uber-would-like-to-buy-your-roboticsdepartment.html?smid=nytcore-ipad-share\&smprod=nytcore-ipad\&_r=1. 